\title{
Asymmetric dependencies in processing phonetic features
}

\author{
PETER D. EIMAS, VIVIEN C. TARTTER, JOANNE L. MILLER, \\ and NANCY J. KEUTHEN \\ Brown University, Providence, Rhode Island 02912
}

\begin{abstract}
A series of four experiments investigated the nature of the dependency relation that exists during the processing of the phonetic features of place and manner of articulation. A speeded classification paradigm was used in which listeners' classification times along these two dimensions were measured under three conditions of variation along the nontarget dimension: (1) no variation, (2) correlated variation, and (3) orthogonal variation. In all four studies, a mutual but asymmetric pattern of interference was found in the orthogonal conditions, in which the magnitude of the interference effect was greater when place of articulation was the target dimension than when manner was the target dimension. In addition, the discriminability of the manner values did not affect the pattern of interference effects. The results were discussed in terms of a concurrent-contingent model of parallel processing wherein it was assumed that feature decisions along either dimension are affected by feature value along the other dimension, but that decisions about place of articulation are more dependent on manner information than manner decisions are on place information.
\end{abstract}

Contemporary theoretical approaches to the perception of speech at the phonetic (segmental) level have been concerned with a description of the processes or stages of analysis that intervene between the acoustic event and the final perception of phonetic segments, for example, the segmental unit [b] in the syllable [ba]. At the very least, the assumption has been made (cf. Pisoni, 1975) that the acoustic signal undergoes a number of transformations (i.e., stages of analysis) which in effect reduce the amount of information in the signal and ultimately yield a set or matrix of distinctive phonetic feature values. These matrices of feature values define the individual segmental units and serve as the basis for perception at this level of linguistic analysis.

There is considerable evidence available from a wide variety of experimental situations in support of the contention that the acoustic signal undergoes some form of distinctive feature analysis. For ex-

This research was supported by Grant HD 05331 from the National Institute of Child Health and Human Development

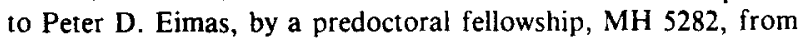
the National Institute of Mental Health to Vivien C. Tartter, and a postdoctoral fellowship, NS 00143 , from the National Institute of Neurological and Communicative Disorders and Stroke to Joanne L. Miller. We would like to express our gratitude to Dr. Alvin M. Liberman for generously making the facilities of the Haskins Laboratories available to us and to Ms. Sheila Polofsky and Ms. Hilary Walker for their assistance in testing subjects and analyzing data. J. L. Miller is now at Northeastern University. Requests for reprints may be addressed to Peter D. Eimas, Department of Psychology, Brown University, Providence, Rhode Island 02912. ample, the existence of a phonetic feature code has been inferred from analyses of perceptual confusions (e.g., Miller \& Nicely, 1955), confusions in shortterm memory (e.g., Wicklegren, 1966), judgments of phonetic similarity (Greenberg \& Jenkins, 1964), the blend errors found in dichotic listening tasks (e.g., Studdert-Kennedy \& Shankweiler, 1970), and the alterations in speech perception resulting from selective adaptation (e.g., Eimas \& Corbit, 1973). There is also substantial evidence that the processes involved in phonetic feature extraction and their manner of operation are markedly the same for a number of different phonetic features. For example, in a recent study directly comparing the processes underlying perception of the phonetic features of place and manner of articulation, Miller and Eimas (1977) found that the labial-alveolar and nasal-stop distinctions were both perceived in a nearly categorical manner, that both yielded similar selective adaptation effects, and finally, that both showed a right-ear advantage of approximately the same magnitude in a dichotic listening task. The latter finding, of course, implicates similar left hemisphere involvement during the analysis of both features. Numerous other reports on the perception of voicing and place distinctions have shown similar processing characteristics (cf. Abramson \& Lisker, 1970; Cooper, 1974a; Eimas \& Corbit, 1973; Lisker \& Abramson, 1970; Mattingly, Liberman, Syrdal, \& Halwes, 1971; Studdert-Kennedy \& Shankweiler, 1970).

In addition to these findings, a number of studies have also provided information directly concerning 
the issue of whether the phonetic features are extracted from the signal independently of one another, as has been suggested by Miller and Nicely (1955), or whether the mechanisms of feature analysis interact in some fashion with one another. Evidence for the latter condition would be obtained if the determination of the value for one feature was influenced by the value of another feature. Somewhat surprisingly, this issue has not been addressed in most models of speech perception, despite its obvious importance and despite the fact that some linguistic theories of distinctive phonetic features have suggested the possibility of dependencies among features that are hierarchical in nature (cf. Chomsky \& Halle, 1968). Be that as it may, there is evidence for dependencies during processing. Smith (1973) has reanalyzed the perceptual confusion matrices of Miller and Nicely (1955) and has shown that the best descriptions of these data require the assumption of dependencies among the features during perception. Furthermore, Smith's analysis revealed that these dependencies are unidirectional or asymmetrical, with the analysis of the information for place of articulation being contingent on the prior analysis of the information for voicing or manner of articulation. Haggard (Note 1) has also argued for a unidirectional dependency between voicing and place of articulation, but in the opposite direction. In accord with Haggard's model, Lisker and Abramson (1970) found that the location of the phonetic boundary distinguishing voiced and voiceless stop consonants varied with the place of articulation value of the stimuli. However, it should be noted that Lisker and Abramson did not test whether there was a dependency in the opposite direction as well, that is, whether the location of the boundaries along the place feature were affected by the value of voicing.

In a recent study that was directly addressed to the problem of dependency relations during the analysis of phonetic features, Sawusch and Pisoni (1974) demonstrated that the identification function for a series of synthetic speech stimuli that varied simultaneously along the acoustic dimensions that signal voicing and place distinctions is best described by the assumption "that the features of place and voicing are not processed independently but rather show a mutual dependency on each other" (p. 181). In addition, Miller (1977) has obtained evidence for a mutual dependency by showing that the location of the voiced-voiceless boundaries for several series of synthetic speech stimuli varied as a function of the feature value for place of articulation and that the labial-alveolar boundaries for similar series of stimuli shifted with changes in the phonetic feature values of voicing and manner of articulation. Furthermore, a number of studies that assessed the effects of selective adaptation on the perception of speech have also provided evidence that the extraction of phonetic features occurs in a dependent manner (e.g., Cooper, 1974b; Cooper \& Blumstein, 1974; Miller \& Eimas, 1976, 1977, Experiment 2).

In summary, there is considerable evidence for dependency effects, that is, processing interactions (cf. Garner \& Morton, 1969), in the analysis of phonetic features from a number of experimental paradigms, although the exact nature of this dependency remains to be determined. It is of interest to note that the nature of the dependency relation may be a function of the experimental paradigm (thereby possibly rationalizing some of the conflicting data) as well as a function of the particular features or the characteristics of the acoustic dimensions underlying these features. That is to say, some combinations of features may be analyzed in a mutually dependent manner, whereas other combinations may be processed in an asymmetric or unidirectional manner and still other combinations may be mutually, but asymmetrically, dependent on each other during processing. The latter possibility can be inferred if, in addition to each feature affecting the processing of the other, there is also a greater effect (i.e., dependency) in one direction than in the other.

The present series of experiments investigated the nature of the dependency that exists between the phonetic features of place and manner of articulation during the processing of speech. The experimental paradigm was a modified two-choice speeded classification procedure (cf. Garner, 1974) that was first used by Wood and Day (1975). This procedure appears to be aptly suited to the purpose of the present experiments, in that it has proven capable of detecting a number of different forms of dependency relations during the processing of auditory information. For example, Wood and Day (1975) found a mutual dependency when one of the acoustic dimensions signaled a phonetic distinction in the initial consonant of a consonant-vowel syllable ([b] vs. [d]) and the other dimension signaled a phonetic distinction in the final vowel ([a] vs. [ae]). This form of dependency has also been found with two nonphonetic dimensions, e.g., pitch and intensity (Wood, 1975a). Asymmetric or unidirectional dependencies have been obtained when one dimension signaled a phonetic distinction (place of articulation) and the other signaled a nonphonetic dimension (pitch) (Wood, 1974), and when both dimensions were nonphonetic in nature (e.g., rise time and intensity) (Blechner, Day, \& Cutting, 1976; and see Pastore, Ahroon, Puleo, Crimmins, Golowner, \& Berger, 1976). Although it is not yet possible to specify the acoustic characteristics of the dimensions that lead to one form of dependency rather than another, it is clear that it is not related simply to the distinction that is often made between phonetic and nonphonetic aspects of acoustic information. 
In the speeded classification procedure, listeners typically classify stimuli that vary along two, binaryvalued acoustic dimensions under three conditions: (1) when only the target dimension varies (the control condition), (2) when both dimensions vary and the variation along the target and nontarget dimensions is perfectly correlated (the correlated condition), and (3) when both dimensions vary and the variation along the target dimension is orthogonal with respect to the variation along the nontarget dimension (the orthogonal condition).

It is possible to infer different forms of dependencies from comparisons of the decision times in the orthogonal and correlated conditions with the decision times in the control condition. Consider first the orthogonal decision times. Independent processing of both features may be assumed if there is no difference between the decision times for the orthogonal and control conditions. Dependencies during feature processing may be inferred if the decision time for the orthogonal condition is longer than the decision time for the control condition. This form of dependency may be said to be mutual if the increment in decision times (i.e., the interference effect) is equal for the two dimensions, whereas, if the interference effect is greater for one dimension than the other, the dependency is mutual, but asymmetric. An interference effect that occurs for only one dimension likewise denotes a dependency during processing, but one that is completely asymmetrical. With regard to the comparison of the decision times in the correlated and control conditions, a redundancy gain (i.e., faster decision times in the correlated condition) can occur under some conditions if the processes of feature analysis are independent parallel events (cf. Lockhead, 1972; Wood, 1975b). A redundancy gain can also occur if the relation between the processes of feature analysis is one of dependency or interaction, particularly if at some level the combination of features is processed as a unitary event or "blob" (cf. Garner, 1974; Lockhead, 1972). An absence of a redundancy gain is quite in accord with an asymmetrical pattern of dependency, ${ }^{1}$ and, as we will propose later, it is also in agreement with a mutual, but asymmetrical, dependency that results from a concurrent-contingent form of multidimensional processing.

\section{GENERAL METHOD}

\footnotetext{
Subjects

Twelve undergraduate and graduate students from Brown University served as volunteer subjects in each of the four experiments. One subject was dropped from Experiment 4 for an excessive number of errors, this listener having contributed more than $31 \%$ of the total number of errors. All of the listeners were native speakers of English with no known hearing loss, and all were paid for their participation.
}

\section{Stimulus Tapes}

In each experiment, there were four consonani-vowel syllables, each of which could be distinguished from the other three in terms of its particular feature values from the two binary-valued phonetic features (dimensions) of place $(P)$ and manner $(M)$ of articulation. ${ }^{2}$ The four stimuli $\left(P_{1} M_{1}, P_{2} M_{1}, P_{1} M_{2}\right.$, and $\left.P_{2} M_{2}\right)$ were combined in various ways to form eight classification tapes: four control tapes, two correlated tapes, and two orthogonal tapes. The stimuli for each of the control tapes were two syllables that differed along one feature, with the other feature held constant at one of its two values, for example, $P_{1} M_{1}$ and $P_{2} M_{1}$. Given the binary nature of the two dimensions, four such control tapes are possible. The correlated tapes consisted of two stimuli that differed from each other along both dimensions, the possible stimuli combinations being $P_{1} M_{3}$ and $P_{2} M_{2}$ or $P_{1} M_{2}$ and $P_{2} M_{1}$. The two orthogonal tapes each contained a different randomization of all four stimuli.

All tapes were prepared with the Pulse Code Modulation System at the Haskins Laboratories. Twelve randomized practice stimuli followed by 64 randomized test stimuli were recorded on one channel of each of the classification tapes. This yielded 32 instances of each syllable in the control and correlated conditions and 16 instances of each syllable in the orthogonal condition. On the second channel of each tape was a series of $50-\mathrm{msec}$ pulses, which were synchronized with the onset of each syllable on the first channel. The interstimulus interval was $4 \mathrm{sec}$.

\section{Procedure}

Each subject listened to two of the four control tapes, the two correlated tapes, and the two orthogonal tapes. Within each of the three conditions, the stimuli on each of the two tapes were classified according to a different dimension. The pair of control tapes presented and the dimension by which each correlated tape was judged were counterbalanced across subjects. The order for presenting the conditions was determined by a balanced Latin square, with the property that each condition followed and preceded every other condition once.

The tapes were played on a Crown 800 tape recorder. Stimuli were binaurally presented to the subjects through Koss Pro 600 AA earphones, at $78 \mathrm{~dB}$ SPL. The pulses on the second channel of the tape recorder triggered a Lafayette voice-activated relay (Model 1604A), which in turn activated a Hunter digital timer (Model 1521) accurate to $1 \mathrm{msec}$. The subject indicated his choice and simultaneously stopped the timer by pressing one of two buttons on a response keyboard. Subjects were instructed to place the index finger of the preferred hand on a neutral, center button, and to move it to a button on the right or left to respond. The response buttons were labeled with values for the appropriate target dimension, with the labels being counterbalanced for position across listeners.

At the beginning of the session, the subject was given a general explanation of the purpose of the experiment and the procedure to be followed, including a description of the entire stimulus set. In addition, the subjects were pretrained to assign to each of the syllables the appropriate feature names. Prior to each condition, the subject was informed of the target dimension and possible stimulus alternatives for that block of trials. In the correlated condition, listeners' attention was not directed to the redundancy in the nontarget values. The subjects were tested individually in a quiet room. In the first three experiments, testing was completed in a single session lasting approximately $1 \mathrm{~h}$. The fourth experiment required three sessions, each about $\mathbf{4 0} \mathrm{min}$ in duration.

\section{EXPERIMENT 1}

The stimuli of Experiment 1 were the four consonant-vowel syllables, [ba], [da], [ma], and [na]. With respect to place of articulation, [ba] and [ma] 
are bilabial consonants and [da] and [na] are alveolar consonants, whereas with respect to manner of articulation, [ba] and [da] are stop consonants and [ma] and [na] are nasal consonants. As noted above, previous research with the speeded classification paradigm (Wood \& Day, 1975) had indicated that a mutual dependency existed between two phonetic dimensions. However, in the Wood and Day study, the two dimensions functioned to change the phonetic quality of different segmental units, i.e., the initial consonant and final vowel of consonant-vowel syllable. In the present study, the two dimensions function to determine the phonetic quality of the same segmental unit, the initial consonant.

\section{Method}

The four stimuli were synthetically produced speech, constructed by means of the parallel resonance synthesizer at the Haskins Laboratories. Previous research has established that all four of the sounds were virtually perfectly identifiable by a group of comparable listeners (Miller \& Eimas, 1977). The two stop consonants, [ba] and [da], were $300 \mathrm{msec}$ in duration, each of which had a $45-\mathrm{msec}$ period of linear formant transitions and a 255 -msec period of steady-state formants. The steady-state frequencies for the first, second, and third formants were 769 , 1,232 , and $2,525 \mathrm{~Hz}$, respectively, and the starting frequency of the first formant was $537 \mathrm{~Hz}$. The starting frequencies for the second and third formants were 921 and $2,018 \mathrm{~Hz}$ for [ba] and 1,695 and $3,363 \mathrm{~Hz}$ for [da], respectively. The nasal consonants [ma] and [na] were constructed by altering their respective stop consonants, [ba] and [da]. First, $60 \mathrm{msec}$ of nasal resonance was added to each of the syllables, $20 \mathrm{msec}$ of which overlapped with the formant transitions, and second, the first-formant transition was made less steep; it had a frequency value of $743 \mathrm{~Hz}$ for the first $25 \mathrm{msec}$ and a value of $769 \mathrm{~Hz}$ for the remaining duration of the syllable. The total stimulus duration of the nasal consonants was $340 \mathrm{msec}$.

The four control tapes were composed of the following combinations of stimuli: (1) [ba] and [da], place varies, with the manner value being stop consonant; (2) [ma] and [na], place varies, with the manner value being nasal consonant; (3) [ba] and [ma], manner varies, with the place value held constant at bilabial; and (4) [da] and [na], manner varies, with a constant place value of alveolar. The two correlated tapes paired bilabial and stop ([ba]) with alveolar and nasal ([da]) and bilabial and nasal ([ma]) with alveolar and stop ([da]). The orthogonal tapes, of course, used all four feature combinations.

\section{Results and Discussion}

The mean reaction times (averaged across feature values, trials, and subjects) are shown in Table 1 for each target condition and each testing condition. Preliminary analysis revealed no reliable effects of order of presentation of the conditions as well as a random distribution of errors, which never exceeded $4 \%$ in any of the six target dimension by condition combinations.

An analysis of variance of the mean decision times for each listener (Subjects by Target Dimensions by Conditions) yielded a significant Conditions effect $[F(2,22)=37.0, p<.001]$ and a significant effect for Targets $[F(1,11)=12.2, p<.01]$. More important, however, was the fact that the Target
Dimensions by Conditions interaction was also highly significant $[F(2,22)=9.5, p<.001]$. Individual comparisons revealed a mutual, but asymmetric, interference effect; that is, the decision times for both orthogonal conditions were reliably greater than the times for their corresponding control groups $(p<$ .01 ), but the magnitude of the effect was greater when place of articulation was the target dimension than when manner of articulation was the target dimension $(p<.01)$.

With regard to the decision times in the correlated condition, there was an overall decrease compared to the mean of the two control times, but further analysis of the interaction effect showed that only the facilitation for the place condition was reliable beyond the .05 level. However, before concluding that a true redundancy gain was obtained, it is necessary to eliminate the possibility that the present listeners used a selective serial processing strategy, that is, that listeners classified the stimuli in the correlated condition in accord with their faster (more discriminable) dimension. A comparison of each listener's mean time in the place correlated conditions with each listener's faster control time revealed no reliable difference $[t(11)=1.7, p<.10]$.

Experiment 1 thus dernonstrated that as with the phonetic features of voicing and place of articulation (e.g., Miller, 1977) a strong dependency exists during the processing of place and manner information. Of particular importance is the additional finding that this dependency, although mutual, is asymmetric: The processing of place of articulation shows a greater dependency on manner information than conversely. Experiment 2 further investigated the dependency in processing place and manner information with stimuli that differed from those of the first experiment along the manner dimension; the manner distinction in Experiment 2 was stop vs. fricative. The values along the place feature were again labial and alveolar.

\section{EXPERIMENT 2}

\section{Method}

The four stimuli were naturally produced consonant-vowel syllables, [ba], [da], [va], and [za]. The syllables [ba] and [da] formed the category stop consonants and the syllables [va] and [za] formed the opposing category of fricatives. Along the dimension of place of articulation, [ba] and [va] have the value of labial

Table 1

Mean Reaction Times in Milliseconds for Each Target Dimension and Condition: Experiment 1

\begin{tabular}{cccc} 
& \multicolumn{3}{c}{ Condition } \\
\cline { 2 - 4 } Dimension & Control & Correlated & Orthogonal \\
\hline Place & 493 & 443 & 675 \\
Manner & 492 & 450 & 544 \\
\hline
\end{tabular}


and [da] and [ra] have the value of alveolar. The irur syllables, produced in a monotone by a male speaker, were digitized, stored, and edited by means of the Haskins Laboratories Pulse Code Modulation System. Each of the syllables was equated for duration, $350 \mathrm{msec}$, and for peak intensity.

Four control tapes were constructed, two of which varied in place of articulation with manner held constant (\{ba] vs. [da] and [va] ss. [za]) and two with variations in manner of articulation with place of articulation held constant ([ba] is. [va] and [da] w. [za]). The two correlated tapes paired either the labial stop [ba] with the alveolar fricative [za] or the alveolar stop consonant [da] with the labial fricative [va]. The two orthogonal tapes consisted of all four syllables, each with a different random order. All other procedural details were the same as those of Experiment 1.

\section{Results and Discussion}

The mean reaction times (again averaged across individual feature values, trials, and subjects) are displayed in Table 2 as a function of the target dimension and testing condition. Preliminary analysis revealed no significant effects of the order in which the conditions were presented. The error rate, which never exceeded $2 \%$ in any of the six combinations of target dimensions and test conditions, varied randomly across conditions.

An analysis of variance of the individual mean reaction times (Subjects by Target Dimensions by Conditions) yielded a significant effect for Conditions $[F(2,22)=28.6, p<.01]$ and for the interaction of Target Dimensions and Conditions $[F(2,22)$ $=7.2, \mathrm{p}<.01]$. Individual analysis revealed that the mean difference of $15 \mathrm{msec}$ between the control and correlated conditions failed to attain statistical significance $(p>20)$, whereas the difference between the control reaction times and orthogonal reaction times (125 $\mathrm{msec}$ ) was highly significant $(p<.001)$. A detailed analysis of the significant interaction revealed that it was virtually entirely attributable to the interference effect which was again mutual, but asymmetric $(p<.01)$ : The processing of manner information was interfered with by the presence of variations in place of articulation and conversely, but the interfering effect of manner information on place decisions was reliably larger than the interfering effects of place information on manner decision.

In summary, then, the pattern of results of Experiment 2 was completely in accord with those of Experiment 1 , in that both studies showed a mutual and asymmetric interference effect and an absence of significant redundancy gains. Experiment 3 was an additional test of the generality of these effects. In this study, the manner distinction was stop consonant vs. glide and the place of articulation distinction was labial vs. velar. In actuality, the place of articulation designating the glide [ja] is alveolarpalatal, which means that the point of major constriction during articulation lies aehind the alveolar ridge and slightly in front of the velar region. Despite [ja] not being truly velar with respect to place, it is
Table 2

Mean Reaction Times in Milliseconds for Each Target Dimension and Condition: Experiment 2

\begin{tabular}{lccc} 
& \multicolumn{3}{c}{ Condition } \\
\cline { 2 - 4 } Dimension & Control & Correlated & Orthogonal \\
\hline Place & 593 & 568 & 749 \\
Manner & 602 & 598 & 695 \\
\hline
\end{tabular}

clearly the case that compared to such sounds as [ba] which is produced at the front of the mouth and [da] which is produced at a middle region, [ya] is produced toward the back of the oral cavity, as is [ga].

\section{EXPERIMENT 3}

\section{Method}

The four sounds of Experiment 3 were the syllables, [ba], [ga], [wa], and [ja], produced by the same speaker and in the same manner as the syllables of Experiment 2. The stimuli werc again digitized, stored, and edited by means of the Pulse Code Modulation System. All stimuli were equated for peak intensity and were $500 \mathrm{msec}$ in duration. Again, four control tapes were made: two in which place varied but manner was held constant ([ba] vs. [ga] and [wa] vs. [ja]) and two in which manner varied and place was held constant ([ba] vs. [wa] and [ga] vs. [ja]). The two correlated tapes consisted of either the labial stop [ba] and velar glide [ja] or the labial glide [wa] and velar stop [ga]. The orthogonal tapes consisted of different random orders of all four syllables. All other procedural details were identical with those of the first two experiments.

\section{Results and Discussion}

Table 3 shows the mean reaction times as a function of target dimension and test condition. Initial analysis revealed no effects of order of presentation of the various tapes. The errors, which never exceeded $1 \%$, were randomly distributed across the various testing conditions and target dimensions.

A Subject by Target Dimensions by Conditions analysis of variance of the individual mean reaction times revealed a significant effect of Target Dimensions $[F(1,11)=5.6, p<.05]$, Conditions $[F(2,22)=20.9, p<.001]$ and the interaction of Target Dimensions and Conditions $[F(2,22)=4.2$, $\mathrm{p}<.05]$. Further analyses of the significant interaction effect revealed a mutual, but asymmetric, interference effect, with manner information again interfering more with the processing of place information than place information interfered with manner decisions. In addition, there was a very

Table 3

Mean Reaction Times in Milliseconds for Each Target Dimension and Condition: Experiment 3

\begin{tabular}{lccc} 
& \multicolumn{3}{c}{ Condition } \\
\cline { 2 - 4 } Dimension & Control & Correlated & Orthogonal \\
\hline Place & 580 & 534 & 738 \\
Manner & 561 & 544 & 657 \\
\hline
\end{tabular}


nearly reliable decrement in the processing of place information in the correlated condition $(p<.06)$, which is to say that there was an asymmetric redundancy gain. However, further analysis showed that it was not possible to reject the hypothesis that this gain was a function of selective serial processing $[t(11)<1, p>.20]$. The listeners did not respond faster to the correlated stimuli in this condition than they did to their faster control dimension.

\section{EXPERIMENT 4}

It is possible to explain an asymmetric or a mutual and asymmetric interference effect in terms of the relative discriminability of the levels of the two target dimensions. The dimension with the more discriminable values should be easier to process when it is relevant and harder to ignore when it is irrelevant compared to the dimension with the less discriminable values (cf. Garner, 1974). Consequently, if, in the present experiments, the place values were less discriminable than the manner values, then, in the orthogonal conditions, longer decision times should occur when place is relevant and manner irrelevant than when manner is relevant and place is irrelevant. ${ }^{3}$ Experiment 4 tested the discriminability hypothesis by systematically varying the acoustic information underlying the manner distinctions in a way that was presumed to decrease the discriminability of the two manner values. If these variations were successful and the discrimination hypothesis correct, then the asymmetry should be eliminated and perhaps even reversed. However, if the asymmetry was not a function of differences in discriminability but, rather, a function of the processing system itself, then variations in the discriminability of the manner values should, within limits, leave the asymmetry unaffected. Obviously, reductions in discriminability beyond the point at which reliable classifications are possible would undoubtedly eliminate the asymmetry, but would at the same time render the experiment meaningless.

\section{Method}

Stimuli. Three sets of synthetic speech stimuli were constructed, each of which varied along the dimensions of manner of articulation (nasal vs. stop) and place of articulation (labial vs. alveolar). The stimuli of Set 1 were the same stimuli used in Experiment 1, with the exception that $20 \mathrm{msec}$ of nasal resonance overlapped the initial $20 \mathrm{msec}$ of formant transitions for the stop consonants as well as for the nasals. The remaining two sets were synthesized by reducing the acoustic differences along the manner dimension, while leaving the acoustic differences along the place dimensions unchanged. Hence, the levels of the place dimension were becoming more discriminable vis à vis the levels of the manner dimension. To accomplish this change in discriminability, the nasal resonance was decreased for the nasal consonants and increased for the stop consonants. In addition, the starting frequency of the first formant was lowered for the nasals and raised for the stops. These manipulations tend to make nasal consonants more stop-like and stop consonants more nasal-like (Miller \& Eimas, 1977). Table 4 shows the durations of the nasal resonance and the first-formant starting frequencies for each of the three sets of stimuli. It should be noted that no changes were made in the starting frequencies of the second and third formants, the acoustic dimensions which were varied in order to signal the two levels of place of articulation.

For each set of stimuli, four control tapes and two orthogonal tapes were prepared in the manner described previously for Experiment 1 . Given the absence of true redundancy gains in earlier experiments and given that this experiment was designed to investigate the source of the asymmetry in the interference effect, it was judged unnecessary to include the correlated conditions.

Procedure. On each of the three daily sessions, each subject received a different set of stimuli, the order of presentation of which was counterbalanced across listeners. Within a session, the order in which the two control tapes and the two orthogonal tapes were presented was determined by a balanced Latin square.

\section{Results and Discussion}

The mean reaction times for Experiment 4 are shown in Table 5. Preliminary analyses again showed no reliable differences as a function of the order in which the conditions were presented. The errors, which never exceeded $3 \%$ in any of the individual cells, occurred more frequently when the target dimension was manner of articulation $[\mathrm{t}(10)=2.4$, $\mathrm{p}<.05]$. Indeed, all of the errors made by the subject who was dropped occurred when the target dimension was manner. It was evidently the case that she had considerable difficulty in classifying the

Table 4

Duration of the Nasal Resonance (in Milliseconds) and the First-Formant $\left(F_{1}\right)$ Starting Frequency Value (in hertz) for the Four Syllables as a Function of Stimulus Set: Experiment 4

\begin{tabular}{|c|c|c|c|}
\hline \multirow[b]{2}{*}{$\begin{array}{c}\text { Stimulus } \\
\text { Set }\end{array}$} & \multirow[b]{2}{*}{ Syllables } & \multicolumn{2}{|c|}{ Acoustic Dimensions } \\
\hline & & $\begin{array}{c}\text { Resonance } \\
\text { Duration }\end{array}$ & $\begin{array}{l}F_{2} \text { Start- } \\
\text { ing Value }\end{array}$ \\
\hline 1 & $\begin{array}{l}\text { [ma] and [na] } \\
{[\mathrm{ba}] \text { and [da] }}\end{array}$ & $\begin{array}{l}60 \\
20\end{array}$ & $\begin{array}{l}734 \\
537\end{array}$ \\
\hline 2 & $\begin{array}{l}{[\mathrm{ma}] \text { and }[\mathrm{na}]} \\
{[\mathrm{ba}] \text { and [da] }}\end{array}$ & $\begin{array}{l}55 \\
25\end{array}$ & $\begin{array}{l}718 \\
562\end{array}$ \\
\hline 3 & $\begin{array}{l}\text { [ma] and [na] } \\
\text { [ba] and [da] }\end{array}$ & $\begin{array}{l}50 \\
30\end{array}$ & $\begin{array}{l}692 \\
588\end{array}$ \\
\hline
\end{tabular}

Table 5

Mean Reaction Times in Milliseconds for Each Target Dimension and Condition as a Function of Stimulus Set: Experiment 4

\begin{tabular}{clccc}
\hline \multirow{2}{*}{$\begin{array}{c}\text { Stimulus } \\
\text { Set }\end{array}$} & Dimension & Control & $\begin{array}{c}\text { Ortho- } \\
\text { gonal }\end{array}$ & $\begin{array}{c}\text { Condition } \\
\text { Differ- } \\
\text { ence* }\end{array}$ \\
\hline \multirow{2}{*}{1} & Place & 452 & 583 & 131 \\
& Manner & 445 & 505 & 60 \\
2 & Place & 467 & 590 & 123 \\
& Manner & 494 & 557 & 63 \\
3 & Place & 454 & 577 & 123 \\
& Manner & 494 & 550 & 56 \\
\hline
\end{tabular}

*Orthogonal minus control 
stimuli along this dimension, and the difficulty was greatly exacerbated by narrowing the acoustic differences between the stimuli. In all other respects, the pattern of errors was random.

In order to determine whether the acoustic changes in the manner dimension yielded less discriminable stimuli, an analysis of variance was performed on the mean decision times for the control conditions alone (Subjects by Target Dimensions by Sets). Decision times increased with a change in the stimulus set $[F(2,20)=3.9, p<.05]$. The times for the first set were shorter than those for the second and third sets, which in turn did not differ from one another. More important, however, was the significant interaction between Target Dimensions and Sets $[F(2,20)=$ 4.24, $\mathrm{p}<.05$ ], which could be attributed nearly entirely to the fact that only the manner targets showed a reliable increment across stimulus sets; the small differences in decision times for the place targets did not even approach significance. It would seem, then, that the acoustic manipulations did in fact alter the discriminability of the manner distinctions, while at the same time the discriminability of the place distinctions was left virtually unchanged.

A second analysis of variance, Subjects by Conditions by Target Dimensions by Sets, revealed a number of reliable effects. First, decision times were significantly slower in the orthogonal condition $[F(1,10)=95.5, p<.001]$. Second, there was a reliable Sets by Target Dimensions interaction $[(2,20)$ $=5.7, \mathrm{p}<.01$ ], indicating that, averaged across conditions, the decision times for manner targets become longer in Sets 2 and 3, whereas the times for the place targets remained constant. Finally, there was a significant Target Dimensions by Conditions interaction $[F(1,10)=25.3 ; p<.001]$. Individual analyses revealed that a significant interference effect was present for both target dimensions, but that the magnitude of this effect was once more greater when place was the target dimension than when manner was the target dimension. Overall, the magnitude of the asymmetry was $66 \mathrm{msec}$, and furthermore, it was quite constant across the three sets of stimuli: 71,60 , and $67 \mathrm{msec}$ for Sets 1,2 , and 3, respectively. Given that we were successfully able to vary the discriminability of the manner values and that the magnitude and the direction of the asymmetry were not related to the relative discriminability of the stimuli, the evidence argues strongly for the case that the asymmetry in the interference effects arises from the way in which these particular dimensions are processed, and not from the specific acoustic properties of the stimuli.

\section{GENERAL DISCUSSION}

The results of the present experiments were not- ably consistent, in that, in all four studies, the processing of information for manner and place of articulation was marked by a mutual, but asymmetric, dependency, as evidenced by mutual, but asymmetric, interference effects. Moreover, despite variations in the particular feature values along these phonetic dimensions and the relative discriminability of the levels within each dimension, the direction of the asymmetry was invariant: The analysis of place of articulation was more dependent on the analysis of manner of articulation than the analysis of manner was on place. Findings of this nature are in accord with a parallel processing model that functions in a concurrent-contingent manner (cf. Turvey, 1973). In this form of processing model, the mechanisms of analysis, while functioning in a temporally overlapping and interactive fashion, ${ }^{4}$ are, to some extent, hierarchically arranged, in that some processes of analysis require the outputs from other analyzers before their own analyses can be completed. To accommodate the present data within this theoretical framework, it is only necessary to assume that, although the mechanisms for the analysis of place and manner information do function to a large extent in a parallel and interactive manner, the analysis of place of articulation requires some form of input from the system that processes manner of articulation.

The present series of experiments was also consistent in its failure to find a true redundancy gain, i.e., faster decision times in the correlated conditions compared to the control conditions that could not be attributed to selective serial processing among other possible explanations. Inasmuch as our instructions for the correlated tasks did not emphasize the correlated nature of the stimuli, as they did in the Wood (1974) and Blechner et al. (1976) studies, we replicated Experiment 1 with instructions that informed listeners about the nature of the stimuli and that further informed them "to try and use the correlation between the two dimensions to help you respond as quickly as you can." Despite this change, a true redundancy gain (or for that matter any form of correlated effect) failed to appear. The absence of a redundancy gain does, we believe, conform to our version of a concurrent-contingent model of parallel processing in that, when classification occurs along the manner dimension, the analysis of manner information will be complete before the analysis of place, thereby precluding any advantage to using the place information. Moreover, when classification is along the place dimension, listeners might utilize the redundant manner information, but if they do so, then their decision times should, in principle, not be reliably faster than the manner control times. It is of interest to note that, in the two instances where a reliable or nearly reliable correlated effect occurred 
(Experiments 1 and 3), the target dimension was place of articulation and the effect could be explained in terms of selective serial processing: Listeners used their faster dimension to classify the stimuli, which for 17 of 24 observers was manner of articulation.

In conclusion, the evidence supports the contention that the processing of manner and place of articulation exhibits a mutual but asymmetric dependency. It is of some interest to note that this form of dependency relation has not been obtained previously with acoustic dimensions, although Pomerantz and Sager (1975) did find a similar dependency relation with visual dimensions. However, despite the uniqueness of this finding, we do not wish to imply that this pattern of dependency is restricted to the processing of phonetic information and consequently is a characteristic of processing in the speech mode (cf. Liberman, Cooper, Shankweiler, \& StuddertKennedy, 1967). Given the numerous recent studies suggesting that the processing of speech and nonspeech acoustic signals is subserved by a common set of auditory analyzers (e.g., Cutting \& Rosner, 1974; Eimas \& Miller, in press; Kuhl \& Miller, 1975), it seems much more likely that a mutual asymmetric dependency is just one of many forms of dependency that exists within the auditory system.

\section{REFERENCE NOTE}

1. Haggard, M. P. The use of voicing information. Speech Synthesis and Perception, Vol. 2, Psychological Laboratory, Cambridge University, 1970. Pp. 1-15.

\section{REFERENCES}

Abramson, A. S.. \& Lisker, L. Discriminability along the voicing continuum: Cross-language tests. In Proceedings of the Sixth International Congress of Phonetic Sciences, Prague, 1967. Prague: Academia, 1970. Pp. 569-573.

Blechner, M. J., Day, R. S., \& Cutring, J. E. Processing two dimensions of nonspeech stimuli: The auditory-phonetic distinction reconsidered. Joumal of Experimental Psychology: Human Perception and Performance, 1976, 2, 257-266.

Сномsky, N., \& Halle, M. The sound pattern of English. New York: Harper \& Row, 1968.

COOPER, W. E. Adaptation of phonetic feature analyzers for place of articulation. Journal of the Acoustical Society of America, 1974, 56. 617-627. (a)

CoOper. W. E. Contingent feature analysis in speech perception. Perception \& Psychophysics, 1974, 16, 201-204. (b)

Cooper, W. E., \& Blumstein, S. E. A labial feature analyzer in speech perception. Perception \& Psychophysics, 1974, 15. 591.600.

Cutting, J. E., \& Rosner, B. S. Categories and boundaries in speech and music. Perception \& Psychophysics, 1974, 16. $564-570$.

Eimas, P. D., \& Corbit, J. D. Selective adaptation of linguistic feature detectors. Cognitive Psychology, 1973, 4, 99-109.

Eimas, P. D., \& Miller, J. L. Effects of selective adaptation on the perception of speech and visual patterns: Evidence for feature detectors. In H. L. Pick, Jr., \& R. D. Walk (Eds.), Perception and experience. New York: Plenum Press, in press.
GARNER, W. R. The processing of information and structure. Potomac, Md: Erlbaum, 1974.

Garner, W. R., \& Morton, J. Perceptual independence: Definitions, models, and experimental paradigms. Psychological Bulletin, 1969, 72, 233-259.

Greenberg, J. J., \& Jenkins, J. J. Studies in the psychological correlates of the sound system of American English. Word, $1964,20,157-177$.

Kuhl, P. A., \& Millek, J. D. Speech perception by the chinchilla: Voiced-voiceless distinction in alveolar plosive consonants. Science, 1975, 190, 69-72.

Liberman, A. M., Cooper, F. S., Shankweller, D. S., \& Studdert-Kennedy, M. Perception of the speech code. Psychological Review, 1967, 74, 431-461.

Lisker, L., \& ABramson, A. S. The voicing dimension: Some experiments in comparative phonetics. In Proceedings of the Sixth International Congress of Phonetic Sciences, Prague, 1967. Prague: Academia, 1970. Pp. 563-567.

Lockmead, G. R. Processing dimensional stimuli: A note. Psychological Review, 1972, 79, 410-419.

Mattingly, I. G., Liberman, A. M., Syrdal, A. K., \& HALWES, T. Discrimination in speech and nonspeech modes. Cognitive Psychology, 1971, 2, 131-157.

Miller, G. A., \& Nicely, P. An analysis of perceptual confusions among some English consonants. Joumal of the Acoustical Society of America, 1955, 27, 338-352.

Miller, J. L. Nonindependence of feature processing in initial consonants. Journal of Speech and Hearing Research, 1977, 20, 519.528 .

Miller, J. L., \& Eimas, P. D. Studies on the selective tuning of teature detectors for speech. Journal of Phonetics, 1976, 4, 119-127.

Miller, J. L., \& Eimas, P. D. Studies on the perception of place and manner of articulation: A comparison of the labialalveolar and nasal-stop distinctions. Journal of the Acoustical Society of America, 1977, 61, 835-845.

Pastore, R. E., Ahroon, W. A., Puleo, J. S., Crimmins, D. B., Golowner, L., \& Berger, R. S. Processing interaction between two dimensions of nonphonetic auditory signals. Journal of Experimental Psychology: Human Perception and Performance, 1976. 2, 267-276.

Plson. D. B. Information processing and speech perception. In G. Fant (Ed.). Speech communications (Vol. 3). New York: Wiley. 1975. Pp. 331-337.

Pomerantz, J. R., \& SAGer, L. C. Asymmetric integrality with dimensions of visual pattern. Perception \& Psychophysics, $1975,18,460-466$

Sawusch, J. R., \& Pisoni, D. B. On the identification of place and voicing features in synthetic stop consonants. Journal of Phonetics, 1974, 2, 181-194.

$\mathrm{S}_{\text {MiTh, }} \mathrm{P}$. T. Feature-testing models and their application to perception and memory for speech. Quarterly Journal of Experimental Psychology, 1973, 25, 511-534.

STUdDeRt-Kennedy, M. \& Shankweiler, D. Hemispheric specialization for speech perception. Journal of the Acoustical Society of America. 1970, 48, 579-594.

TURVEY. M. T. On peripheral and central processes in vision: Inferences from an information-processing analysis of masking with patterned stimuli. Psychological Review, 1973, 80, 1-52.

WICKFLGREN, W. A. Distinctive features and errors in short-term memory for English consonants. Joumal of the Acoustical Society of America, 1966, 39, 388-398.

WINER, B. J. Statistical principles in experimental design. New York: McGraw-Hill, 1971.

Wood. C. C. Parallel processing of auditory and phonetic information in speech discrimination. Perception \& Psychophysics. 1974, 15, 501-508.

Wood, C. C. Auditory and phonetic levels of processing in speech perception: Neurophysiological and informationprocessing analyses. Journal of Experimental Psychology: Human Perception and Performance, 1975, 1, 3-20. (a) 
Wood, C. C. A normative model for redundancy gains in speeded classification: Application to auditory and phonetic dimensions in speech discrimination. In F. Restle, R. M. Shiffrin, N. J. Castellan, H. Landman, \& D. B. Pisoni (Eds.), Cognitive theory (Vol. 1). Potomac, Md: Erlbaum, 1975. Pp. 55-77. (b)

Wood, C. C., \& DAY. R. S. Failure of selective attention to phonetic segments in consonant-vowel syllables. Perception \& Psuchophysics, 1975, 17, 346-350.

\section{NOTES}

1. A number of studies (Blechner et al., 1976; Pastore et al., 1976, Experiment 5; Wood, 1974) have shown an asymmetric interference effect and a redundancy gain. One explanation of this combination of effects is "that subjects have some degree of freedom about the kinds of processing that they use in different task conditions" (Blechner et al., 1976, p. 259). However, it is also possible that the use of an inappropriate error term may have resulted in a Type I error and the erroneous conclusion that there was a significant redundancy gain in the Wood and Blechner et al. studies. In both cases, the error term involved an interaction of Conditions by Trials. Inasmuch as the ANOVA design included the random variable of subjects, a more appropriate error term is the Conditions by Subjects interaction (cf. Winer, 1971). Although a proper analysis was used in the Pastore et al. study, it should be noted that they did not test for whether or not the redundancy gain could be attributed to selective serial processing or other factors (cf. Wood, 1974).
2. It should be recognized that when we refer to binary-valued dimensions it is with reference to stimuli within a specific experiment, inasmuch as place of articulation may assume more than two values and manner of articulation does not constitute a simple unidimensional continuum.

3. Despite differences in discriminability along the two dimensions, it is possible to obtain equal decision times in the two control conditions. The levels of both dimensions could be sufficiently discriminable such that reaction time measures will not detect differences in discriminability because of floor effects. However, when the task is made more difficult by the presence of variable irrelevant information and processing is slowed, then differences attributable to discriminability will have the opportunity to be exhibited.

4. We have used the term interactive inasmuch as we believe that the mutual portions of the interference effects are not simply a function of some form of limited capacity or shared attention or response mapping. If the interaction (i.e., interference effects) were attributable to any of these factors, then there would be no reason to expect that in obtaining identification functions the assignment of feature values to stimuli near the phonetic boundary would be influenced by the particular feature values along other dimensions, as in fact they are (cf. Miller, 1977; Sawusch \& Pisoni, 1974).

(Received for publication May 24, 1977; revision accepted October $15,1977$. 